\title{
The WATCH solar X-ray burst catalogue ${ }^{\star}$
}

\author{
N. Crosby ${ }^{1,2}$, N. Lund ${ }^{3}$, N. Vilmer ${ }^{1}$, and R. Sunyaev ${ }^{4}$ \\ 1 DASOP URA2080, Observatoire de Paris, Section d'Astrophysique, 5 Place J. Janssen, 92195 Meudon, France \\ 2 LPCE, UPR 4010 du CNRS, conventionnée avec l'Université d'Orléans, 3A avenue de la Recherche Scientifique, 45071 Orléans \\ la Source Cedex 2, France \\ 3 Danish Space Research Institute, Juliane Maries Vej 30, 2100 Copenhagen, Denmark \\ ${ }^{4}$ Space Research Institute, Profsoyuznaja 84/32, 117296 Moscow, Russia
}

Received July 22; accepted November 18, 1997

\begin{abstract}
The WATCH experiment aboard the GRANAT satellite provides observations of the Sun in the deka-keV range covering the years 1990 through mid-1992. An introduction to the experiment is given followed by an explanation of how the WATCH solar burst catalogue was created. The different parameters listed for each burst is given and are furthermore explained.
\end{abstract}

Key words: catalogs — sun: activity; flares; X-rays, gamma rays

The Danish Wide Angle Telescope for Cosmic Hard X-rays (WATCH) experiment was launched aboard the Russian GRANAT satellite on the 1st of December 1989 and is composed of four WATCH units. One of these units has the Sun in its field-of-view and observes in the deka-keV range with approximately $6.5 \mathrm{~s}$ time resolution. WATCH is based on the rotation-modulation-collimator (RMC) principle. The WATCH instrument contains two independent detectors, one based on a NaI- the other on a CsIscintillator. The direction of the incoming X-rays can thus be derived from the modulation of the detected signal as a function of the rotation phase of the collimator grids. The modulation patterns are used in determining the positions and strengths of the X-ray sources in the field-ofview. In this work observations from the NaI scintillator have been used. For more details on the WATCH experiment see Lund (1985) and for the solar observations see Crosby (1996) and Crosby et al. (1997).

The WATCH solar burst catalogue consists of 1551 flares and was created by systematically going through the approximately 2.5 years of count rate time profile ob-

Send offprint requests to: N. Crosby

* The catalogue is available in electronic form only at the CDS via anonymous ftp to cdsarc.u-strasbg.fr (130.79.128.5) or via http://cdsweb.u-strasbg.fr/Abstract.html servations. WATCH observations are grouped in dumps associated to telemetry periods.

To be characterized as being of solar origin a burst had to pass the following four selection criteria (see Crosby et al. 1997, for more detail):

1. The modulation pattern must correspond to the solar one.

2. A signature in the GOES data has to be observed (a classified GOES flare or a significant flux enhancement). The corresponding WATCH peak is not necessarily at the same time as that of the GOES peak.

3. The peak count rate of the WATCH event has to be above the three sigma WATCH background level. This criterion eliminates 5 very small events.

4. The event must not be significantly contaminated by particles.

The WATCH solar burst catalogue is divided into months (Tables 2 through 27) and for each month the events are listed in chronological order (one line per event). The information listed in each line (from left to right) is as follows:

For each burst the dump number (DN) where the burst is recorded and the day (DAY) when the burst began is listed. Thereafter the total peak count rate $\left(P_{\mathrm{t}}\right)$ in counts per bin and the duration in seconds $\left(T_{\mathrm{acc}}\right)$ of the bin are indicated. This information refers to observations recorded in the low-energy channel of WATCH. The time accumulation (s/bin) for the count rate is based on the rotation velocity of the modulation grid/scintillator system which is slightly variable with time but which is recorded.

The peak time $\left(T_{\text {peak }}\right)$, start time $\left(T_{\text {start }}\right)$ and end time $\left(T_{\text {end }}\right)$ in UT of the event are indicated as hh:mm:ss, where "NN:NN:NN" implies that the information is missing.

The value of the background $(B)$ (c/bin) which is indicated is converted to the time accumulation of the bin. For each burst the background is determined as an average over a duration much longer than the flare (usually 


\begin{tabular}{cccccccccccccc}
\hline \multicolumn{10}{c}{ OCTOBER 1990 } \\
\hline DN & DAY & $\begin{array}{c}P_{\mathrm{t}} \\
(\mathrm{c} / \mathrm{bin})\end{array}$ & $\begin{array}{c}T_{\text {acc }} \\
(\mathrm{s} / \mathrm{bin})\end{array}$ & $\begin{array}{c}T_{\text {peak }} \\
(\mathrm{UT})\end{array}$ & $\begin{array}{c}T_{\text {start }} \\
(\mathrm{UT})\end{array}$ & $\begin{array}{c}T_{\text {end }} \\
(\mathrm{UT})\end{array}$ & $\begin{array}{c}B \\
(\mathrm{c} / \mathrm{bin})\end{array}$ & $\mathrm{H}$ & $\mathrm{d}$ & o & AR & GOES & H $\alpha$ \\
\hline 203 & 06 & 1666 & 6.72 & $16: 58: 54$ & $16: 58: 14$ & $17: 02: 28$ & 1286 & 0 & 0 & 0 & 6300 & $\mathrm{C} 2.3$ & 1 \\
203 & 06 & 5623 & 6.72 & $18: 41: 15$ & $18: 38: 01$ & $19: 10: 06$ & 1286 & 0 & 0 & 0 & 6304 & $\mathrm{C} 4.5$ & 1 \\
203 & 06 & 3496 & 6.72 & $22: 08: 00$ & $22: 06: 53$ & $22: 10: 47$ & 1292 & 0 & 0 & 0 & 6309 & $\mathrm{C} 2.2$ & 1 \\
203 & 06 & 1959 & 6.72 & $23: 50: 36$ & $23: 50: 09$ & $23: 54: 03$ & 1292 & 0 & 0 & 0 & 0 & $\mathrm{~N}$ & 0 \\
203 & 07 & 2091 & 6.72 & $03: 34: 21$ & $03: 33: 27$ & $03: 34: 54$ & 1292 & 0 & 0 & 0 & 6300 & $\mathrm{~N}$ & 1 \\
\hline
\end{tabular}

larger than half an hour) taken either after or before the flare.

An increase in count rate above background in the high-energy channel $(\mathrm{H})$ in association with the solar burst is symbolized as: 1 for yes, 0 for no. " $n$ " implies that some particle noise is observed in the high-energy channel, but not at the peak time of the burst. 27 events were observed with a significant signal in the high-energy channel.

In the next two columns dead-times (d) and over-flow (o) are indicated. Overflow effects are seen in the lowenergy count rate data and refer to situations where the 16-bit count capacity of the WATCH data registers are exceeded, so that the register resets to zero. This has been corrected for in the catalogue by adding to the peak count rate $\left(P_{\mathrm{t}}\right) N^{*} 65536$ counts per bin, where $N$ refers to the number of times the counter resets. Dead-time effects are seen for large flares and observed as a dip in the highenergy data. Dead-time effects are not corrected for in the catalogue.

In the case where it is possible to identify an associated active region (AR) its number is indicated in the next column (see Crosby et al. 1997, for the explanation of how this was done). The GOES flare identification is listed if one exists in Solar Geophysical Data (SGD) comprehensive reports, otherwise " $N$ " is indicated ("ng" implies that no GOES data exists). Approximately $45 \%$ of the WATCH events could be identified with GOES flares in SGD comprehensive reports. The last column indicates that an $\mathrm{H}_{\alpha}$ flare was observed at the same time as the WATCH event (1 for yes, 0 for no). See Crosby et al. (1997) for an explanation of how the $\mathrm{H}_{\alpha}$ association was done.
A list of the periods during which solar observations with WATCH are available (WATCH ON-TIME) is found at the beginning of the catalogue (Table 1). It is presented by month, for example:

\begin{tabular}{|c|c|c|c|c|}
\hline & & $\mathrm{MARCH}$ & 1990 & \\
\hline \multirow[t]{3}{*}{ DN } & start & start & end & end \\
\hline & day & time & day & time \\
\hline & & (UT) & & (UT) \\
\hline 068 & 03 & 13:40:00 & 03 & $15: 08: 00$ \\
\hline 070 & 04 & 13:16:00 & 05 & $23: 32: 00$ \\
\hline 073 & 08 & 14:03:00 & 08 & $17: 55: 00$ \\
\hline 086 & 27 & $22: 14: 29$ & 27 & $23: 11: 42$ \\
\hline
\end{tabular}

More information concerning the WATCH solar burst catalogue and access to the data can be requested by contacting:

\section{Norma Bock Crosby \\ DASOP URA2080, Observatoire de Paris, Section d'Astrophysique, \\ 5 Place J. Janssen, 92195 Meudon, France \\ Electronic mail address: crosby@obspm.fr}

\section{References}

Crosby N., 1996, Ph.D. thesis, Paris VII University, France Crosby N., Vilmer N., Lund N., Sunyaev R., 1997, A\&A (in press)

Lund N., 1985, in: Culhane J.L. (ed.), X-ray Inst. Astron. SPIE 597,95 